\title{
Beam offset due to beam-beam interactions at a warm linear collider
}

\author{
Nicolas Delerue, ${ }^{*}$ Toshiaki Tauchi, ${ }^{\dagger}$ and Kaoru Yokoya ${ }^{\ddagger}$ \\ High Energy Accelerator Research Organization (KEK), 1-1 Oho, Tsukuba Science City, 305-0801 Ibaraki-ken, Japan
}

(Received 15 November 2004; published 21 November 2005)

\begin{abstract}
At a warm linear collider the short time interval at which bunches will pass near each other in the interaction region may lead to significant alteration of the bunches positions. In this paper we quantify the intensity of this effect and show that it can be addressed by a fast intrapulse feedback system.
\end{abstract}

DOI: 10.1103/PhysRevSTAB.8.112801

PACS numbers: $41.75 . \mathrm{Ht}$

\section{BEAM-BEAM INTERACTION AND BEAM BLOWUP AT A WARM LINEAR COLLIDER}

In a linear collider, near the interaction point (IP) after the final magnet the two beams are not any more shielded from each other by the beam pipe. Thus if the outgoing beam has been deflected vertically at the interaction point it will induce a vertical deflection of the incoming beam, leading to a loss of luminosity (see Fig. 1) and an increasing displacement of the beam along the train.

Let the vertical offset of the $k$ th electron (positron) bunch at the IP in units of the rms beam size $\sigma_{y}$ be $\Delta_{k}^{(-)}$ $\left(\Delta_{k}^{(+)}\right)$and define the relative offset by $\Delta_{k}=\Delta_{k}^{(-)}-\Delta_{k}^{(+)}$ (we assume that the two beam have roughly the same size). Let the offsets without beam-beam interaction be $\Delta_{k, 0}$. Then, the offsets with interaction are obtained successively by [1]

$$
\begin{gathered}
\Delta_{k}=C \sum_{l=\max (1,(k-N))}^{k-1} F\left(\Delta_{l}\right)+\Delta_{k, 0} \\
C \equiv\left(\frac{\sigma_{x}}{\sigma_{z} \phi}\right)^{2} D_{x} D_{y}
\end{gathered}
$$

where $D_{x(y)}$ is the horizontal (vertical) disruption parameter, $\sigma_{x(z)}$ the horizontal (longitudinal) bunch size, $\phi$ the crossing angle, $N$ the number of bunches that a given bunch sees on its journey from the last quad to the IP. The form factor $F(\Delta)$ is defined by

$$
F(\Delta)=\frac{\gamma\left(\sigma_{x}+\sigma_{y}\right)}{N_{p} r_{e}} \theta_{y}(\Delta),
$$

where $r_{e}$ is the classical electron radius, $N_{p}$ the number of particles in a bunch, $\gamma$ the particle energy in units of rest mass, and $\theta_{y}(\Delta)$ the beam deflection angle when the beam offset is $\Delta$, as shown in Fig. 2. $\left[F(\Delta) \approx \Delta\right.$, when $D_{y} \ll 1$ and $|\Delta| \ll 1$.]

\footnotetext{
*Electronic address: n.delerue1@physics.ox.ac.uk Now at the John Adams Institute for Accelerator Science at the University of Oxford.

Electronic address: toshiaki.tauchi@kek.jp

‡Electronic address: kaoru.yokoya@kek.jp
}

This number $N$ can be calculated using the following formula:

$$
N=\frac{2 \times \text { distance between IP and last quad }}{\text { bunch spacing } \times c}
$$

(here $c$ is the velocity of the beam taken as the velocity of the light in the calculations below).

An interesting point to note in this formula is that the offset is independent of the location at which bunch $k$ and $l$ cross each other. This happens because two different effects compensate each other. On the one hand the further away from the IP the crossing happens, the bigger the distance between bunches $k$ and $l$ is and thus the smaller the deflection angle of the bunch $k$ will be. But one the other hand, the distance traveled by the bunch after receiving this kick will be longer, thus making the offset at the IP bigger.

The simulations presented in this paper have been done using CAIN [2] with two sets of parameters, one close to the proposed parameters of the GLC (Global Linear

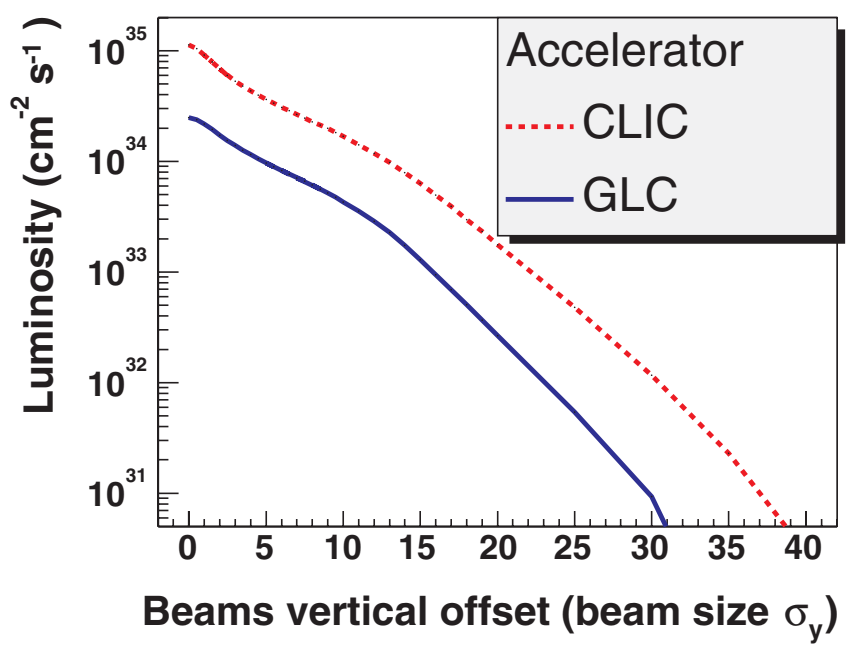

FIG. 1. (Color) Total luminosity delivered as a function of the vertical offset of the beams at the interaction point. The horizontal unit, $\sigma_{y}$, is the vertical size of the beam (a few nanometers). The parameters used for this simulation are those of the GLC and of CLIC as given in Table I. Perfect crab crossing (or head-on collisions have been assumed). 


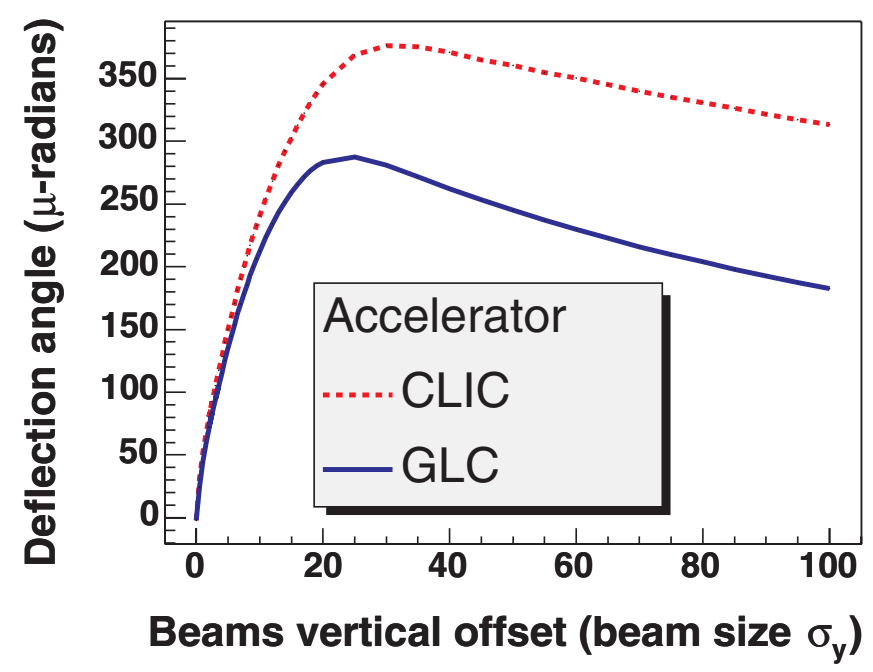

FIG. 2. (Color) Beam deflection angle $\left(\theta_{y}\right)$ as a function the bunch offset at the interaction point $\left(\Delta_{k}\right)$. The parameters used for this simulation are those of the GLC and of CLIC as given in Table I.

Collider) and the other closer to the Compact e Linear Collider (CLIC) specifications. The parameters' values used for these studies are adopted from the ITRC report [3] and are summarized in Table I.

\section{EFFECT OF THE CROSSING ANGLE AND THE OTHER BEAM PARAMETERS ON THE BEAM BLOWUP}

The two parameters that have the biggest influence on the beam blowup are the crossing angle and the number $(\mathrm{N})$ of outgoing bunches seen by an incoming bunch. Figure 3 shows how the blowup [simulated as described by Eq. (1)] varies when the crossing angle varies from the smallest proposed value $(7 \mathrm{mrad})$ to a much less challenging value (30 mrad) and the Table II indicates the vertical offset of

TABLE I. Beam parameter values (at $500 \mathrm{GeV}$ ) used from the blowup simulations adopted from the ITRC report [3].

\begin{tabular}{|c|c|c|}
\hline \multirow{2}{*}{$\begin{array}{l}\text { Set } \\
\text { Energy }(\mathrm{GeV})\end{array}$} & GLC/NLC & C CLIC \\
\hline & 243 & 202 \\
\hline$\sigma_{x}(\mathrm{~nm})$ & 243 & 202 \\
\hline$\sigma_{y}(\mathrm{~nm})$ & 3 & 1.2 \\
\hline$\sigma_{y^{\prime}}(\mu \mathrm{rad})$ & 27 & 24 \\
\hline$\sigma_{z}(\mu \mathrm{m})$ & 110 & 35 \\
\hline$D_{x}$ & 0.16 & 0.04 \\
\hline$D_{y}$ & 13.1 & 6.4 \\
\hline$C$ [Eq. (2)] & 0.209 & 0.0213 \\
\hline$\phi($ crossing angle) $(\mathrm{mrad})$ & $7(20)$ & 20 \\
\hline Bunch spacing (ns) & 1.4 & 0.67 \\
\hline$L^{*}$ (distance between IP and last quad) $(\mathrm{m})$ & 3.5 & 4.3 \\
\hline$N$ (bunches) [Eq. (4)] & 16 & 42 \\
\hline
\end{tabular}

the last bunch of the train. (It is assumed that $\Delta_{k, 0}$ is the same for all bunches of a train.)

As one can see on this figure, for an initial offset of $1 \sigma_{y}$, even with a crossing angle of $7 \mathrm{mrad}$ the maximum beam offset at the GLC due to the beam blowup does not exceed $3 \sigma_{y}$. For a crossing angle of $10 \mathrm{mrad}$ or more the beam offset remains below $1.6 \sigma_{y}$. At CLIC the shorter bunch spacing increases the blowup effect. It can reach $23.9 \sigma_{y}$ for a crossing angle of $7 \mathrm{mrad}$ and $5.3 \sigma_{y}$ for a crossing angle of $10 \mathrm{mrad}$. For wider crossing angle, the blowup remains below $2 \sigma_{y}$. If the initial offset is bigger $\left(5 \sigma_{y}\right.$ or $10 \sigma_{y}$ ) then the final offset increases but the increase, which is related to the form factor shown on Fig. 2, is less than linear and the normalized offset ( $\left.\frac{\text { bunch offset }}{\text { initial offset }}\right)$ is smaller, as shown on Fig. 4).

To minimize the blowup effect it is better to locate the last quad closer to the IP but this is not desirable for the detector performance. Instead, it may be possible to keep the incoming and outgoing beam in separate pipes until they are very close from the IP, thus shielding them for each other's influence and reducing $\mathrm{N}$ (and thus the blowup).

On Fig. 5 one can see the influence of $\mathrm{N}$ on the blowup. The offset of the last bunch of the train for various values of $\mathrm{N}$ is given in Table III. As shown previously, at very small crossing angle (7 mrad), the beam blowup is very important and thus the unshielded length has a strong influence on the total blowup. With such crossing angle reducing the unshielded length by $20 \mathrm{~cm}$, from 4.2 to $4.0 \mathrm{~m}$ can reduce the vertical offset of the last bunch of the train by more than $1.0 \sigma_{y}$ for both the GLC and CLIC. At higher crossing angle (20 mrad), the beam blowup is much lower and thus the unshielded length has a smaller influence on the blowup.

\section{BLOWUP AND FAST FEEDBACK SYSTEM}

Ground motion and other sources of vibrations may induce random changes in the beam offset from train to train. To reduce the luminosity lost due to this offset fast intrapulse feedback systems have been proposed for the GLC [and the Next Linear Collider (NLC)] [4-6] to correct the beam offset by measuring the offset of the deflected outgoing pulses with a beam position monitor (BPM) and correcting the incoming pulses with a kicker. After initial correction a delay loop acts to prevent the system from forgetting the correction already applied.

As the beam blowup also results in a beam offset, the fast intrapulse feedback systems can also deal with it.

Mathematically the effect $\left(d_{k}\right)$ of the feedback system described in [4] on bunch $k$ can be described as follows:

$$
\begin{aligned}
& \text { if }(k<b) \text { then } \delta_{k}=0 \quad \text { and } d_{k}=0 \text {, } \\
& \text { if }(k \geq b) \text { then } \delta_{k}=g * F\left(\Delta_{k-b}\right) \text {, }
\end{aligned}
$$



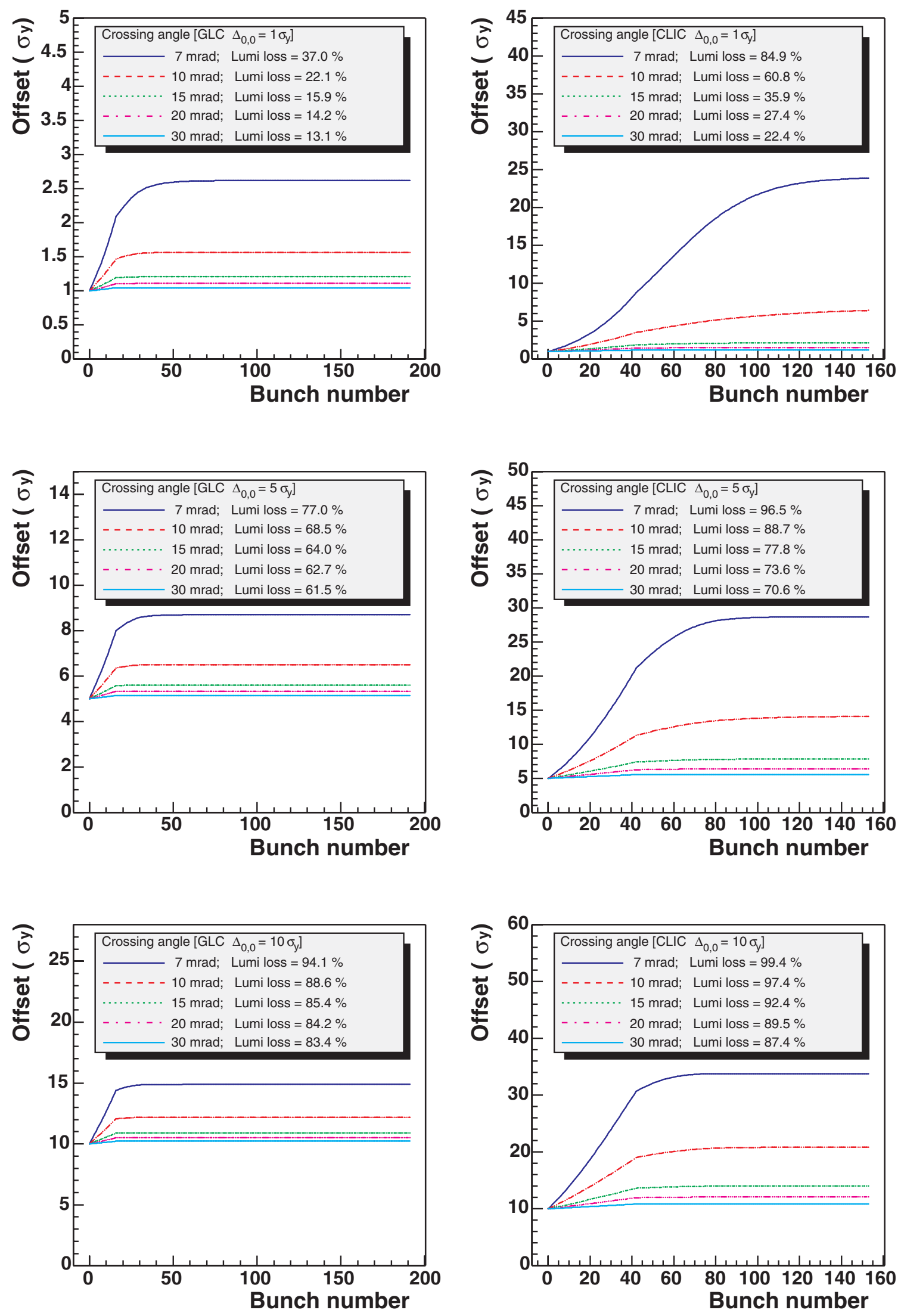

FIG. 3. (Color) Beam blowup as a function of the bunch number for an initial beam offset of $1 \sigma_{y}$ (upper plots), $5 \sigma_{y}$ (middle plots) or $10 \sigma_{y}$ (lower plots) for different values of the crossing angle. The left column correspond to simulations with the GLC (NLC) parameters and the right column correspond to simulations with the CLIC parameters. 
TABLE II. Vertical offset (expressed in beam size $\sigma_{y}$ ) of the last of the train for various crossing angles (the other parameters have the values mentioned in Table I).

\begin{tabular}{lcccccc}
\hline \hline $\begin{array}{l}\text { Crossing angle } \\
(\phi)(\mathrm{mrad})\end{array}$ & \multicolumn{2}{c}{$1 \sigma_{y}$ offset } & \multicolumn{2}{c}{$5 \sigma_{y}$ offset } & \multicolumn{2}{c}{$10 \sigma_{y}$ offset } \\
GLC/NLC & CLIC & GLC/NLC & CLIC & GLC/NLC & CLIC \\
\hline 7 & 2.6 & 23.9 & 8.7 & 28.7 & 14.9 & 33.8 \\
10 & 1.6 & 6.4 & 6.5 & 14.1 & 12.2 & 20.8 \\
15 & 1.2 & 2.2 & 5.6 & 7.8 & 10.9 & 14.0 \\
20 & 1.1 & 1.5 & 5.3 & 6.3 & 10.5 & 12.1 \\
30 & 1.0 & 1.2 & 5.1 & 5.6 & 10.2 & 10.9 \\
\hline \hline
\end{tabular}
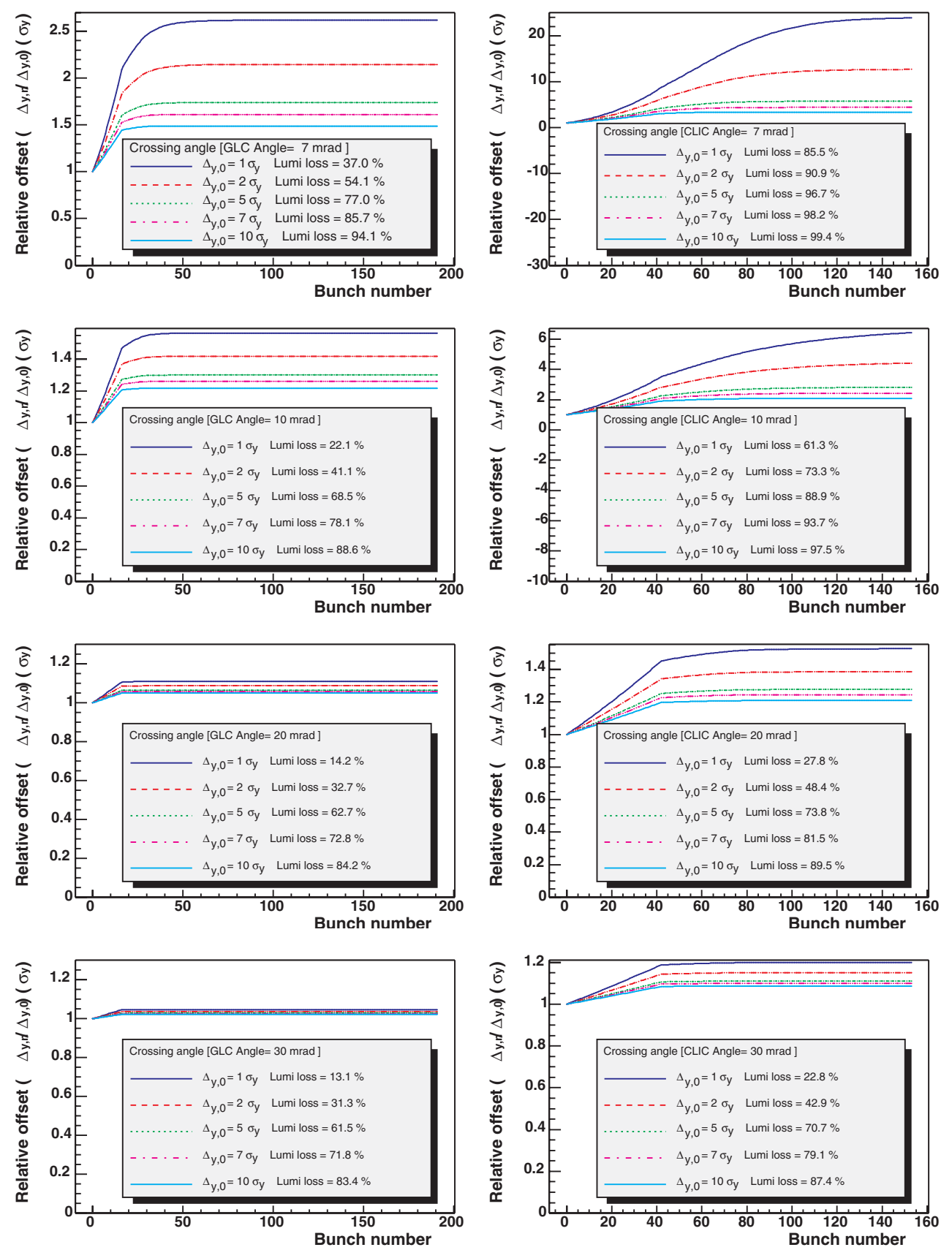

FIG. 4. (Color) Normalized beam blowup as a function of the bunch number for a crossing angle of (from top to bottom) 7, 10, 20, and $30 \mathrm{mrad}$ for different values of the initial beam offset. The normalization is done by dividing the actual offset of a given bunch by the initial beam offset. 

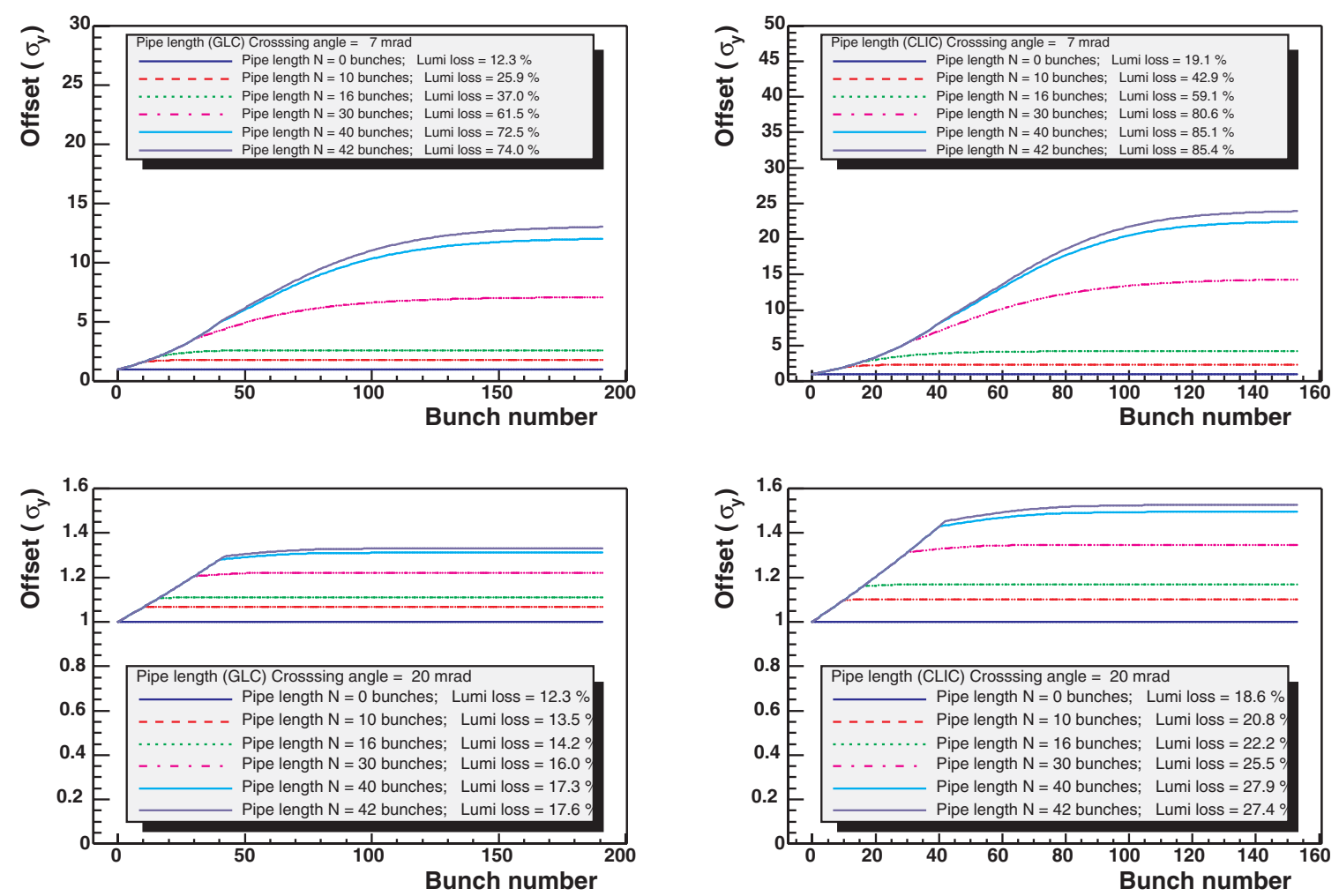

FIG. 5. (Color) Beam blowup as a function of the number of outgoing bunches seen by an incoming bunch for an initial beam offset of $1 \sigma_{y}$ for a crossing angle of $7 \mathrm{mrad}$ (upper plots) and $20 \mathrm{mrad}$ (lower plots).

$$
\text { and } d_{k}=\delta_{k}+d_{k-b}
$$

where $\Delta_{k, 0}$ is the initial offset of the $k$ th bunch (as defined above), $F\left(\Delta_{k}\right)$ is the relative angle with which bunch $k$ was deflected ( $F$ is shown on Fig. 2), $b$ is the latency of the system (that is the distance separating the BPM of the feedback system from the kicker plus the electronic latency, expressed in number of bunches), $g$ is the gain of the system (typically 0.6 for the GLC in normal conditions), and $\delta_{k}$ is the correction specific to bunch $k$ to which the correction $d_{k-b}$ (memorized by the delay loop) is added to give $d_{k}$, the total correction to be applied.
This correction $d_{k}$ is directly subtracted from $\Delta_{k}, 0$ before computing the effect of the beam-beam blowup as shown on Eq. (1). The electronic circuit of the system described in [4] is shown on Fig. 6.

The effect of the beam blowup on the performance of these fast intrapulse feedback systems is shown on Fig. 7. As one can see when the crossing angle is wide and thus blowup is not too intense (10 mrad and more for the GLC, $20 \mathrm{mrad}$, and more for CLIC), the fast feedback system can correct the beam blowup whereas for smaller crossing angles the blowup drives the feedback system into oscillations between overcorrection and undercorrection.

TABLE III. Vertical offset (expressed in beam size, $\sigma_{y}$ ) of the beams at the end of the train for different values of $\mathrm{N}$ (number of outgoing bunches seen by an incoming bunch) for two different crossing angle values (the other parameters have the values mentioned in Table I). $\mathrm{N}$ is proportional to the length during which the two beam are not shielded from each other in the interaction region (IR).

\begin{tabular}{lrrrrrr}
\hline \hline \multirow{2}{*}{ Number of bunches seen } & \multicolumn{2}{c}{ IR length $L^{*}(\mathrm{~m})$} & \multicolumn{2}{c}{$7 \mathrm{mrad}$} & \multicolumn{2}{c}{$20 \mathrm{mrad}$} \\
& GLC/NLC & CLIC & GLC/NLC & CLIC & GLC/NLC & CLIC \\
\hline 0 & $0 \pm 0.2$ & $0 \pm 0.1$ & 1 & 1 & 1 & 1 \\
10 & $2.1 \pm 0.2$ & $1.0 \pm 0.1$ & 1.8 & 2.3 & 1.1 & 1.1 \\
16 & $3.4 \pm 0.2$ & $1.6 \pm 0.1$ & 2.6 & 4.2 & 1.1 & 1.2 \\
30 & $6.3 \pm 0.2$ & $3.0 \pm 0.1$ & 7.1 & 14.3 & 1.2 & 1.3 \\
40 & $8.4 \pm 0.2$ & $4.0 \pm 0.1$ & 12.0 & 22.4 & 1.3 & 1.5 \\
42 & $8.8 \pm 0.2$ & $4.2 \pm 0.1$ & 13.0 & 23.9 & 1.3 & 1.5 \\
\hline \hline
\end{tabular}




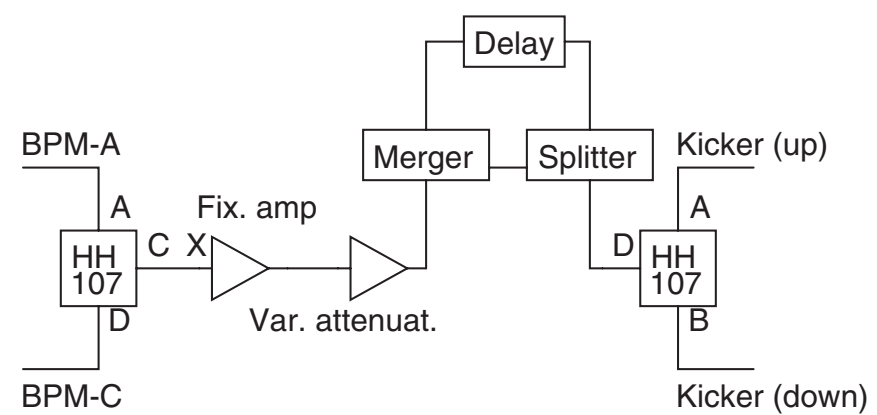

FIG. 6. Circuit of the FEATHER [4] fast intrapulse feedback system.
These oscillations come from the delay between the time at which a correction is applied and the time at which the BPM measures the effects of this correction. Thus after correcting for a given effect the system still measure "uncorrected" bunches. This delay is induced by the time of flight from the kicker to the BPM and by the latency of the electronics used. Figure 8 shows that these oscillations appear regardless of the position of the fast feedback system (or the latency induced by the electronics), but their intensity increases when the system is located further away from the IP.
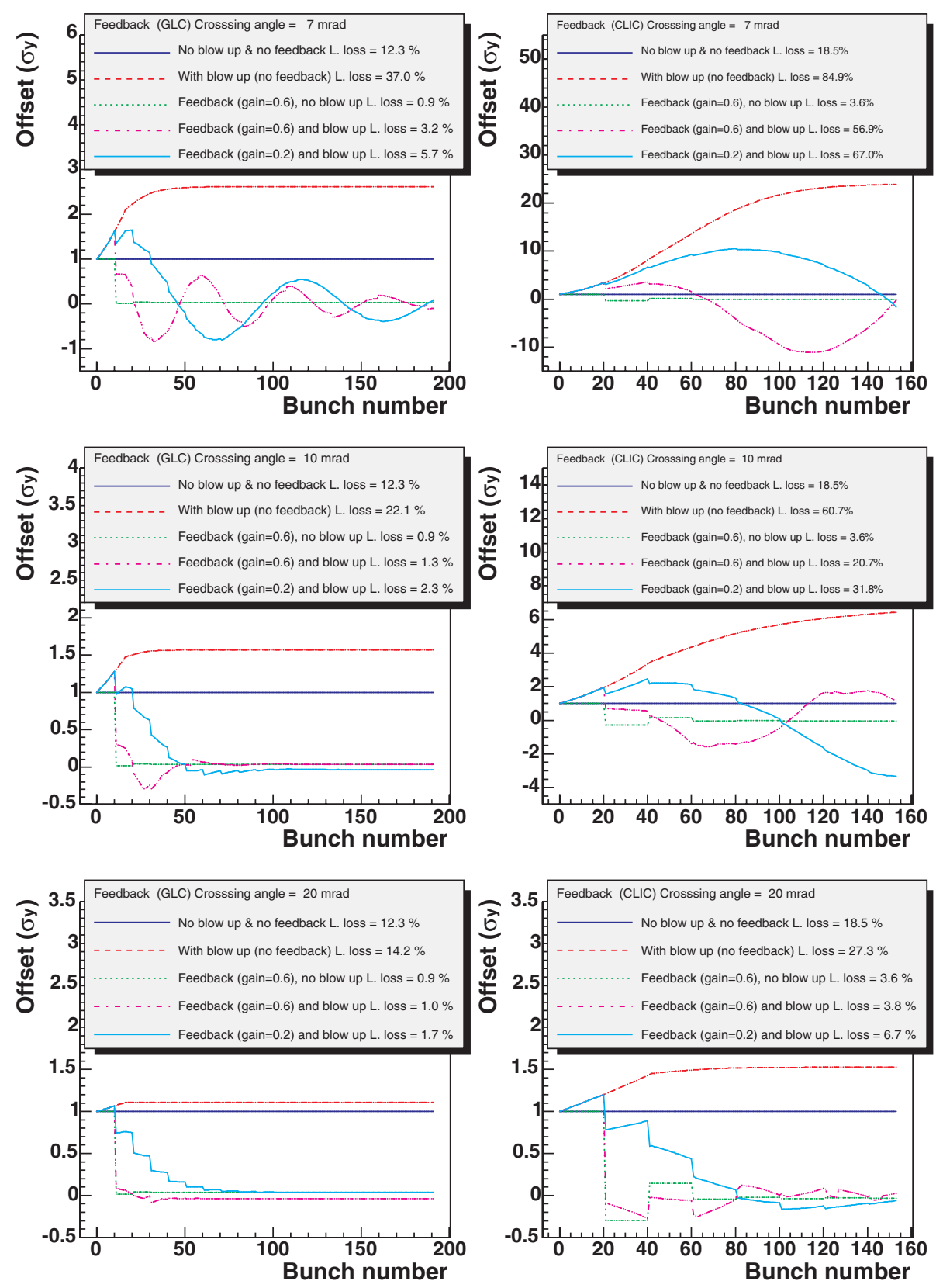

FIG. 7. (Color) Effect of the fast intrapulse feedback system on the beam blowup for different crossing angles for the GLC (left column) and CLIC (right column) for an initial beam offset of $1 \sigma_{y}$. The feedback system is assumed to be located near the last quad. 

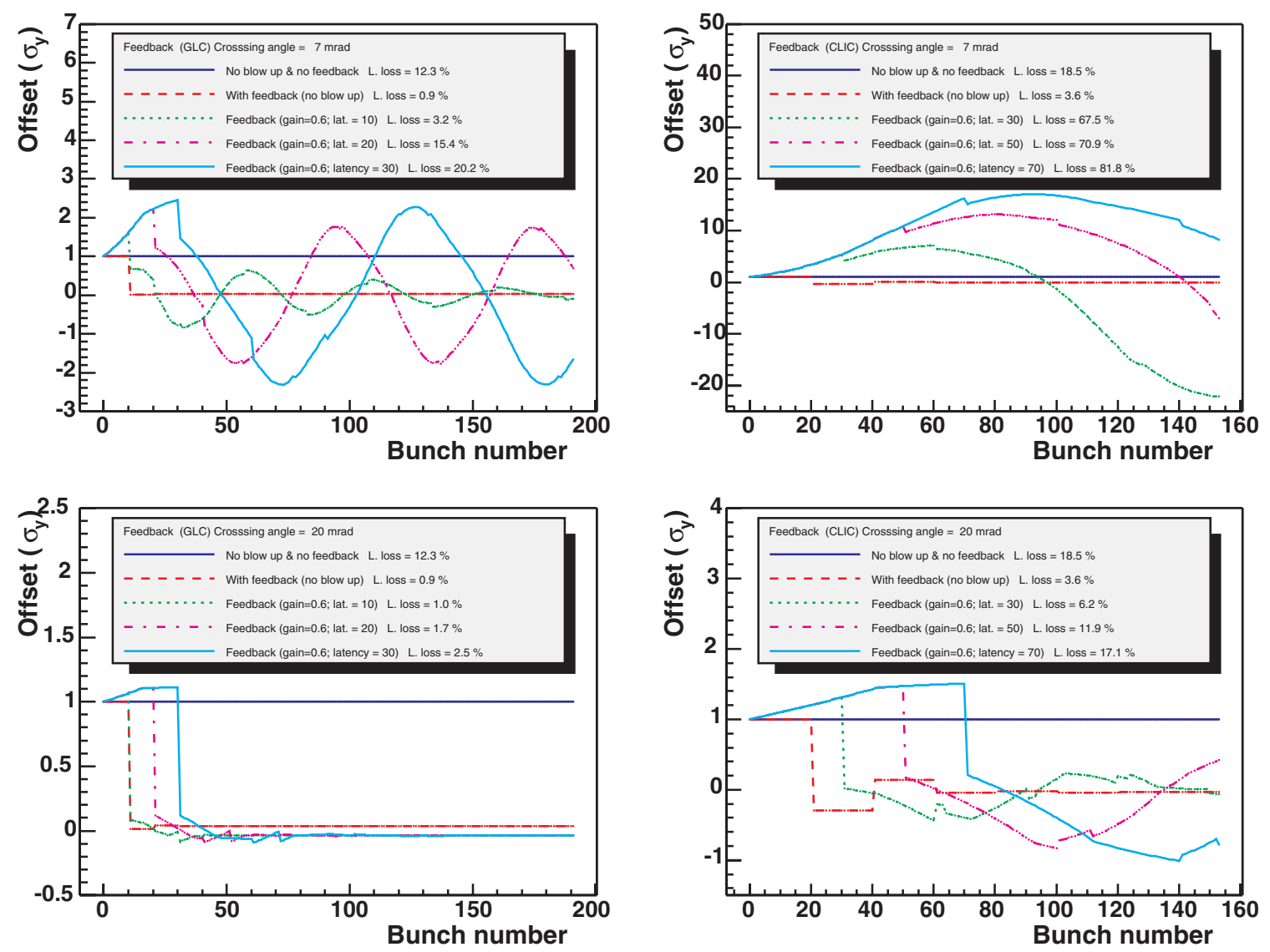

FIG. 8. (Color) Effect of the fast intrapulse feedback system on the beam blowup for different total latency (i.e. position) of the feedback system for the GLC and CLIC for an initial beam offset of $1 \sigma_{y}$. A feedback system latency of 10 (30) for the GLC corresponds to a feedback system located $\sim 2 \mathrm{~m}(\sim 6 \mathrm{~m})$ away from the IP. Latencies of 30 (70) for CLIC corresponds to a feedback system located $\sim 2.8 \mathrm{~m}(\sim 5.8 \mathrm{~m})$ away from the IP.

The delay loop of the fast feedback system (see Fig. 6) addresses some of the problems created by the system's latency but it slows the capacity of the system to adapt to changing conditions such as those created by the beam blowup.

Thus to avoid the oscillations in the fast feedback system, one needs to add a second component to the correction predicted by the feedback system. The intensity of this second component must be directly proportional to the measured bunch position and should not be included in the delay loop. A modified feedback system including this second component is shown on Fig. 9.

Mathematically this new circuit requires the addition of a new term $\epsilon_{k}$ to Eq. (7) to compute the correction $c_{k}$ :

$$
\begin{aligned}
& \text { if }(k<b) \text { then } \delta_{k}=d_{k}=\epsilon_{k}=c_{k}=0, \\
& \text { if }(k \geq b) \text { then } \delta_{k}=g * F\left(\Delta_{k-b}\right), \\
& \text { and } \epsilon_{k}=g_{b} * F\left(\Delta_{k-b}\right), \\
& \text { and } d_{k}=\delta_{k}+d_{k-b},
\end{aligned}
$$

$$
\text { and } c_{k}=d_{k}+\epsilon_{k}
$$

where $c_{k}$ is the correction to be applied and $g_{b}$ is a proportionality coefficient (the gain of the feedback branch). The only difference between $\delta_{k}$ and $\epsilon_{k}$ is that the later is not included in the recursive term $d_{k}$.

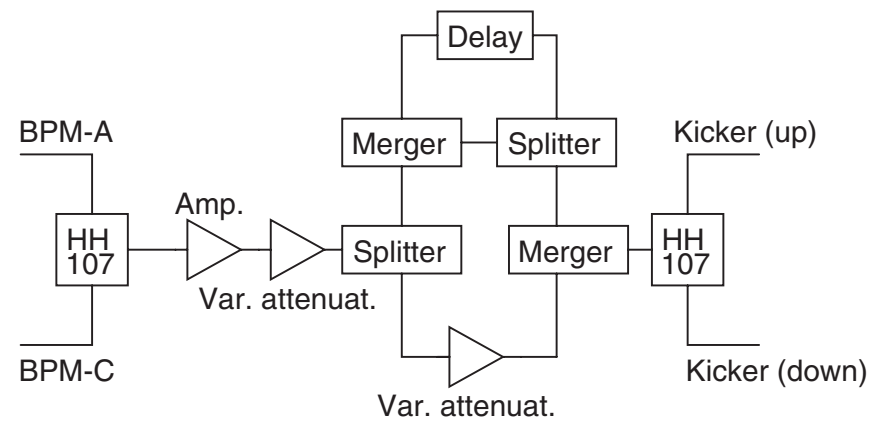

FIG. 9. Modified fast intrapulse feedback system to avoid the oscillations created by the beam blowup. Compared to the FEATHER [4] circuit one extra branch has been added that directly takes the beam position signal and bypasses the delay loop. 

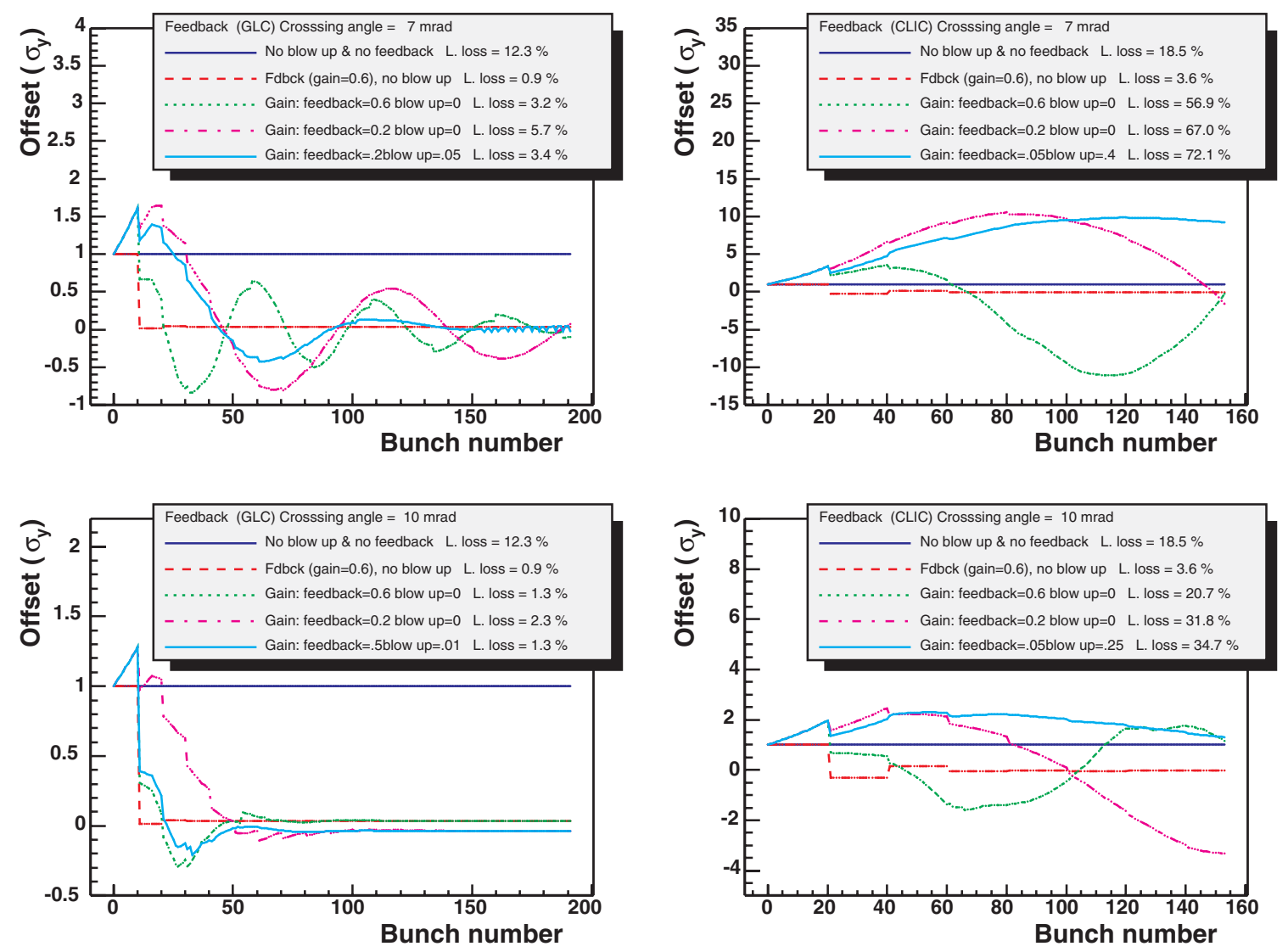

FIG. 10. (Color) Effect of the modified fast intrapulse feedback system on the beam blowup for different crossing angles for the GLC (left column) and CLIC (right column) for an initial beam offset of $1 \sigma_{y}$. The feedback system is assumed to be located near the last quad. The fast feedback system used here has been modified as shown on Fig. 9.

The performances of this modified circuit are shown on Fig. 10. The gains used for these numerical simulations are $g=0.2$ and $g_{b}=1$ for the GLC with a crossing angle of $7 \mathrm{mrad}, g=0.05$ and $g_{b}=18$ for CLIC at the same crossing angle. With a crossing angle of $10 \mathrm{mrad}$ these values become $g=0.5 / g_{b}=0.16$ (GLC) and $g=$ $0.05 / g_{b}=11.25$ (CLIC). These values have been obtained by tuning the system to minimize the luminosity loss. The ratio between these two values reflects the contribution of the beam blowup to the total beam offset. As one can see this modification cancels or reduces the luminosity loss due to the blowup.

\section{BLOWUP CORRECTION BASED ONLY ON THE FIRST BUNCH MEASUREMENT}

As the offset of each bunch of the train is only affected by events (ground motion, transverse long-range wakefield, compensation error of beam loading,...) that are known once the first bunch of the train reaches the IP, the correction to be applied to each bunch can be predicted once the offset of the first bunch is known. This property could be used to design a system that would compute the correction to be applied to each bunch based mainly on the measurement of the offset of the first bunch of the train. To cope with residual ground motion, a simple feedback system (without delay loop) must be added to this system. As the correction to be applied as a function of the bunch offset is not linear such system would have to be tuned for a given offset at which it would perform the best. The simple feedback loop would then perform the second order adjust-

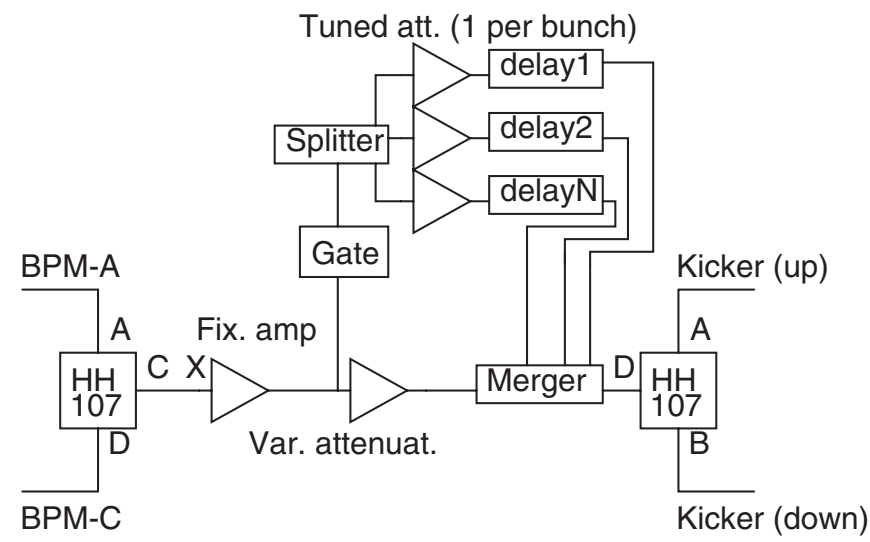

FIG. 11. This circuit uses mainly the information coming from the first bunch to correct the whole train. To correct the residual components of the beam offset a simple feedback loop is also included. 

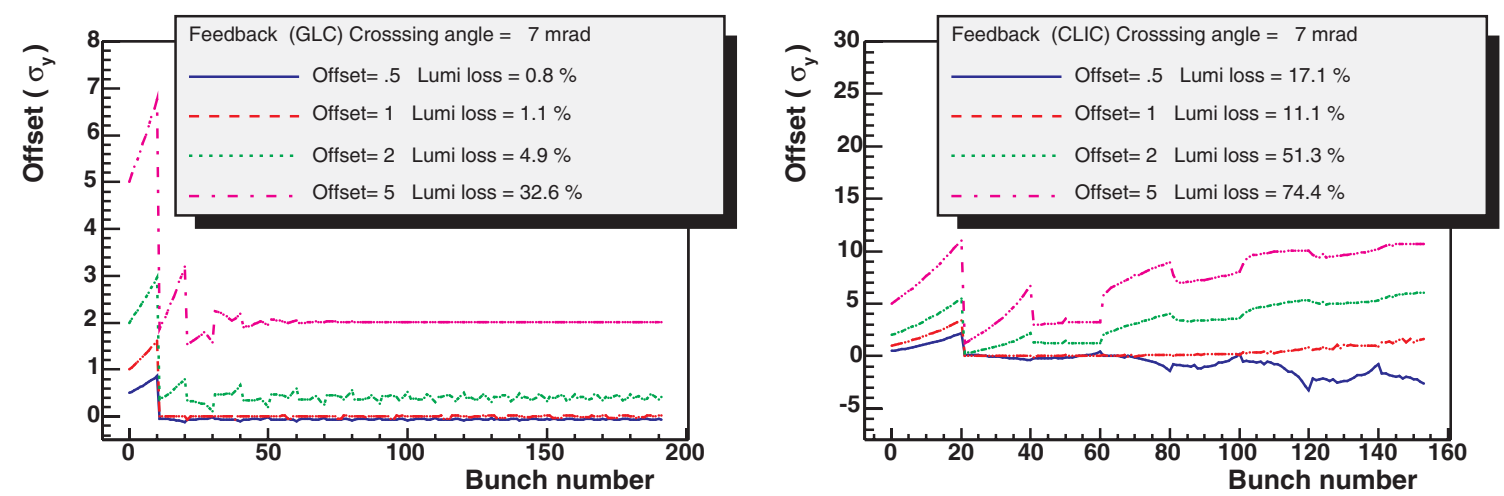

FIG. 12. (Color) Correction of the beam offset using the circuit shown on Fig. 11. The gain used for the simple feedback loop is 0.5 for the GLC and 0.7 for CLIC. The gains used in the other part of the circuit have been tuned so that the beam offset becomes null if the initial offset was 1 .

ments to remove the residual beam offset. By using switches it would be possible to switch between different sets of gains tuned for different initial offsets. Figure 11 shows an example of circuit (without switch) that could be used to implement such system. Figure 12 shows the performances of such a circuit.

As one can see the performances of such a system are rather attractive but the number of attenuators and wires required would be proportional to the number of bunches times the number of switches needed (as each of the bunches would require its own circuit). This huge number of wires needed might be a problem as it would increase the amount of dead material in the detector.

It is important to stress that in the two models presented in the previous section and the one in this section only analog electronics have been used but by the date at which the linear collider will be built very fast digital electronics will probably be available allowing a better correction of the end of the train.

\section{CONCLUSION}

The beam-beam interactions at a warm linear collider such as the GLC or CLIC will create a blowup of the beam, especially at low crossing angle. If the crossing angle is wide enough then the blowup will be corrected by the fast intrapulse feedback system. For smaller crossing angle the blowup will interfere with the feedback system but minor modifications of the feedback system will remove these interferences and correct the beam blowup.

\section{ACKNOWLEDGMENTS}

One of the authors (N.D.) would like to thank the Japanese Society for the Promotion of Science for funding his stay in Japan under Contract No. P02794.

[1] Kaoru Yokoya and Pisin Chen, in Proceedings of the USCERN School on Particle Accelerators, Hilton Head Island, SC, 1990; Lect. Notes Phys. 400, 415 (1992).

[2] P. Chen, G. Horton-Smith, T. Ohgaki, A. W. Weidemann, and K. Yokoya, Nucl. Instrum. Methods Phys. Res., Sect. A 355, 107 (1995).

[3] International Linear Collider Technical Review Committee, Second Report No. SLAC-R-606, 2003.

[4] Nicolas Delerue, physics/0305017.

[5] D. Schulte, physics/0008128.

[6] P. N. Burrows, physics/0112080. 